\title{
Philippe Desan, Montaigne. Une biographie politique
}

\section{Concetta Cavallini}

\section{(2) OpenEdition}

\section{Journals}

\section{Édition électronique}

URL : http://journals.openedition.org/studifrancesi/1121

DOI : 10.4000/studifrancesi. 1121

ISSN : 2427-5856

\section{Éditeur}

Rosenberg \& Sellier

\section{Édition imprimée}

Date de publication : 1 novembre 2014

Pagination : 584

ISSN : 0039-2944

\section{Référence électronique}

Concetta Cavallini, « Philippe Desan, Montaigne. Une biographie politique », Studi Francesi [En ligne], 174 (LVIII | III) | 2014, mis en ligne le 01 novembre 2014, consulté le 18 septembre 2020. URL : http:// journals.openedition.org/studifrancesi/1121; DOI : https://doi.org/10.4000/studifrancesi. 1121

\section{Ce document a été généré automatiquement le 18 septembre 2020.}

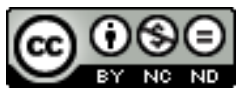

Studi Francesi è distribuita con Licenza Creative Commons Attribuzione - Non commerciale - Non opere derivate 4.0 Internazionale. 


\title{
Philippe Desan, Montaigne. Une biographie politique
}

\author{
Concetta Cavallini
}

\section{RÉFÉRENCE}

PHILIPPE DESAN, Montaigne. Une biographie politique, Paris, Odile Jacob, 2014, pp. 727.

1 Cet imposant volume, Montaigne. Une biographie politique, présente les hypothèses et le résultat des dernières recherches (souvent encore en cours) de Philippe Desan sur l'auteur des Essais. La définition de 'politique' permet de placer Montaigne et sa vie dans un contexte social, dans un réseau de connaissances, d'ambitions personnelles, d'ambitions de groupe social: dans l'univers de Montaigne, Philippe Desan inclut l'ensemble hétérogène de la société de la Renaissance, «ordres, clans, familles, clientèles et corps constitués [...] clercs, nobles, robins, bourgeois» (p. 593). Le parcours individuel de Montaigne croise des événements historiques qui en expliquent la parabole; mais surtout, c'est cette lecture en filigrane proposée par Desan qui peut vraiment faire la lumière sur les Essais: «dans un livre qui clame sans cesse sa consubstantialité avec un corps de chair et de sang, il est difficile de faire la part des choses entre l'homme public et l'homme privé qui se confondent parfois [...]» (p. 29), voilà l'idée qui a déclenché cette analyse.

2 Le volume, dont le texte est supporté par de nombreuses notes critiques (pp. 599-676) et par une Bibliographie très détaillée (pp. 677-708) partagée en trois sections (Sources manuscrites, Sources imprimées et Bibliographie critique) vante une écriture fluide et attirante, qui fascine le lecteur et le conduit à la découverte d'un Montaigne nouveau. Le parcours proposé par Desan est partagé en deux parties: «Ambitions» (pp. 35-313) et «Pratiques» (pp. 315-574). Dans la première, on suit la carrière de Montaigne à travers ses années de formation, en partant de l'histoire de la famille Eyquem, elle aussi soumise à toute une série de malentendus et de fausses croyances. Car une des problématiques principales de la biographie de Montaigne est de savoir faire le tri 
entre les nombreux mythes qu'on a construits au cours des siècles autour de lui et de sa famille, comme celui de l'ascendance juive de sa mère. $\mathrm{Ph}$. Desan rappelle qu'il n'y a aucune preuve de cela et que le silence de Montaigne sur sa mère, qui a fait couler beaucoup d'encre, viendrait plutôt de la volonté de cacher les origines roturières d'Antoinette de Louppes (dont la famille, d'origine espagnole, était dans le commerce du pastel) et donc d'affirmer sa propre noblesse.

3 Toute la première partie de la vie de Montaigne, de sa formation au collège de Guyenne à l'amitié avec La Boétie puis à l'édition de ses ouvrages après la mort de son ami, sa familiarité avec le marquis de Trans, qui fut à la base de son rôle politique et des récompenses d'honneur (l'Ordre de Saint Michel, par exemple) qu'on lui octroya, jusqu'à la véritable 'construction' de la première édition des Essais, que Desan définit comme «le meilleur résumé de carrière possible» (p. 307), de manière à obtenir la faveur du roi Henri III, rencontré en 1580 au château de Saint-Maur (la cour avait fui Paris à cause d'une épidémie de peste), tout paraît calculé, agencé, recomposé en vue d'une carrière politique.

4 La deuxième section commence par le voyage en Italie de 1580-81 et présente l'hypothèse, supportée par une analyse précise et pleine de preuves documentaires, selon laquelle Montaigne, par son voyage, visait à obtenir une ambassade dans la péninsule. Desan insère sa lecture 'politique' du voyage en Italie dans une présentation du domaine diplomatique à la Renaissance, une présentation détaillée qui débouche presque dans des pages d'histoire culturelle, décrivant la nature du corps diplomatique, les caractéristiques sociales et personnelles qu'un ambassadeur devait posséder, mais aussi la différence entre un ambassadeur ordinaire, un ambassadeur extraordinaire et les chargés de mission intérimaires, sans oublier la logique de recrutement que le roi appliquait (p. 324 et suiv.). La relecture de Philippe Desan concerne donc la «nature» (p. 318) même du voyage en Italie, à la lumière des ambitions politiques de Montaigne pendant les années 1575-1580. Les liens entre les La Chassaigne (famille de la femme de Montaigne) et les La Rochepozay (famille de l'ambassadeur en poste à Rome à l'arrivée de Montaigne) sont aussi analysés, ainsi que le différend entre Henri III et Grégoire XIII sur la nomination de Paul de Foix comme archevêque de Toulouse et la concession de la citoyenneté romaine à Montaigne; sur toutes ces questions, Desan fournit des documents de première main, comme par exemple les lettres du cardinal de Côme.

5 La première partie du Journal (celle où Montaigne avait encore des espoirs de carrière) et la deuxième (où il savait déjà qu'il ne pouvait plus espérer devenir ambassadeur) sont totalement différentes dans leur ton et dans leur nature. Après la désillusion, Montaigne s'adonne au plaisir des bains et des cures, à l'apprentissage de l'italien. En échange de l'ambassade envolée, le roi lui donne la Mairie de Bordeaux. Entre 1581 et 1585, Montaigne joue donc un rôle important dans sa Guyenne natale, surtout dans une tentative de réconciliation entre le Béarnais et le roi de France, avant que sa carrière politique ne sombre dans l'oubli après 1585 («sans pour autant être totalement arrêtée», p. 453). Le troisième livre des Essais, sorti en 1588, porte les traces de la désillusion de Montaigne face aux honneurs et aux charges honorifiques. Les dernières années de sa vie (1588-1592) furent des années de «marginalisation» (chapitre X), pendant lesquelles Montaigne de consacra entièrement à l'otium littéraire et à la révision de son ouvrage.

6 Une petite section de conclusion, qui a pour titre «Post Mortem» (pp. 575-596), explique comment, après la mort de Montaigne en 1592, sa carrière politique a sombré dans 
l'oubli à l'avantage de son côté littéraire: ce sont les ouvrages critiques posthumes, avant tout ceux de Marie de Gournay, qui ont eu «pour effet de transformer Montaigne en littérateur et en philosophe» (p. 582). Desan offre un aperçu complet de ce parcours à travers les siècles, pour conclure par une affirmation qui paraît banale, mais qui n'est pas à la mode «à une époque [la nôtre] où tout doit converger vers le moment présent» (p. 591): avant d'être un maître de la pensée universelle, comme on a voulu longtemps le considérer, Montaigne fut nécessairement un auteur de son temps. Il était donc grand temps de procéder à une historicisation de sa pensée.

7 Essai académique ou livre de divulgation, cette biographie politique de Montaigne? L'un et l'autre. Le volume de Philippe Desan s'adresse aux spécialistes de Montaigne mais aussi aux curieux, aux amateurs, à ceux qui veulent avoir une idée de comment fonctionnait la société de la Renaissance. Ouvrage polyvalent donc, cette dernière étude de Philippe Desan: livre de référence pour les études montaignistes, mais aussi ouvrage sur la culture, les mentalités, la sociabilité de toute une époque. Le mérite de l'auteur a été de partager de manière naturelle avec le lecteur son savoir mais aussi son amour et sa passion pour l'auteur des Essais. 